\title{
Phytochemical constituents and preliminary toxicity evaluation of leaves from Rourea induta Planch. (Connaraceae)
}

\author{
Milena Kalegari ${ }^{1 *}$, Marilis Dallarmi Miguel ${ }^{1}$, Josiane de Fátima Gaspari Diass ${ }^{1}$ Ana Luísa Lacava \\ Lordello $^{2}$, Cristina Peitz de Lima ${ }^{1}$, Cristina Mayumi Sasaki Miyazaki ${ }^{1}$, Sandra Maria Warumby \\ Zanin', Maria Christina dos Santos Verdam¹, Obdulio Gomes Miguel ${ }^{1}$
}

\author{
${ }^{1}$ Department of Pharmacy, Federal University of Paraná, ${ }^{2}$ Department of Chemistry, Federal University of Paraná
}

\begin{abstract}
Most active plants are toxic at high doses and it is therefore important to investigate the preliminary toxicity of plant extracts. The Rourea induta species is a potential drug with no phytochemical or biological studies registered in the literature. Thus, a phytochemical study and a toxicity analysis of the ethanolic extract obtained from the leaves of Rourea induta Planch., Connaraceae, was run. A long chain hydrocarbon, $n$-tetracosane, and four flavonoids were identified: quercetin, and three glycosylated derivates, quercetin-3- $O$ - $\alpha$-arabinofuranoside, quercetin-3- $O$ - $\beta$-xyloside and quercetin-3-O $\beta$-galactoside. This is the first time these have been isolated in this species. The structures were elucidated by ${ }^{13} \mathrm{C} \mathrm{NMR}$, ${ }^{1} \mathrm{H}$ NMR, UV and IR spectroscopy. The toxicity evaluation of extracts was performed by the brine shrimp method and determination of hemolytic activity. The samples demonstrated no toxic potential by the analyzed methods.
\end{abstract}

Uniterms: Rourea induta/phytochemistry. Connaraceae/phytochemistry. Flavonoids. $N$-tetracosane. Plant extracts/toxicity.

\begin{abstract}
A maioria das plantas ativas é tóxica em doses elevadas, portanto, é importante a investigação da toxicidade preliminar dos extratos das plantas. A espécie Rourea induta é uma droga potencial que não apresenta estudo fitoquímico ou biológico descrito na literatura. Assim, um estudo fitoquímico e análises toxicológicas foram realizados com o extrato etanólico obtido das folhas de Rourea induta Planch., Connaraceae. Foram obtidos um hidrocarboneto de cadeia longa, $n$-tetracosano, e quatro flavonóides, quercetina e três derivados glicosilados, quercetina-3- $O$ - $\alpha$-arabinofuranosideo, quercetina-3- $O$ - $\beta$-xilosideo e quercetina-3- $O$ - $\beta$-galactosideo. Esta é a primeira vez que estes compostos são isolados nesta espécie. As estruturas foram elucidadas por espectroscopia de $\mathrm{RMN}-{ }^{13} \mathrm{C}, \mathrm{RMN}-{ }^{1} \mathrm{H}$, ultravioleta e infravermelho. A avaliação da toxicidade dos extratos foi analisada pelo método da Artemia salina e atividade hemolítica. Nenhuma das amostras testadas apresentou um potencial tóxico pelos métodos analisados.
\end{abstract}

Unitermos: Rourea induta/fitoquímica. Connaraceae/fitoquímica. Flavonóides. $N$-tetracosano. Extrato de plantas/toxicidade.

\section{INTRODUCTION}

The Connaraceae family comprises approximately 16 genera among 300 to 350 species distributed throughout tropical areas of the world; that consists of the angiosperms characterized by ligneous, trees or shrub species. In America, five genera can be found: Bernardinia, Cnestidium, Connarus, Pseudoconnarus and Rourea (Forero, 2007).

\footnotetext{
*Correspondence: M. Kalegari. Departamento de Farmácia, Universidade Federal do Paraná. Av. Prefeito Lothário Meissner, 632 - Jardim Botânico 80210-170 - Curitiba - PR, Brasil. E-mail: milena.kalegari@gmail.com
}

Connaraceae family native species are spread mainly in the Amazon, and the shrubby species are common in "cerrado" (Brazilian ecosystem composed of short trees and shrubs) and are similar to legume species (Lorenzi, Souza, 2005). In "cerrado", the family is represented by seven shrubby-arboreal species distributed around the genera Rourea and Connarus (Lenza et al., 2008). The cerrado is the second biggest Brazilian and Latin American biome, and has many potential medicinal species that have not yet been subject to scientific study (Recor, 2009). 
Rourea is a pantropical genus and comprises 42 species and 19 varieties; the Amazon is its dispersion center (Forero, 1976; Fonseca, Proença, 2002). The Rourea induta Planch. species is characterized by small trees and shrubs that grow to a maximum height of 4 meters. It bears greenish and densely ramified young shrubs. Its cork is widely known for the treatment of Rheumatism (Fonseca, Proença, 2002).

Some important biological activities have been described for species of the Connaraceae family, whereas the Rourea induta species has no registered studies in the literature. Phytochemical and biological activity studies are a starting point contributing toward the valuable detection of the species and its isolated compounds.

Most active plants are toxic at high doses and it is thus important to investigate the preliminary toxicity of plant extracts. To achieve a toxicological evaluation, the brine shrimp method and hemolytic activity determination techniques have been employed for different groups (Oliveira et al., 2009). Against this background the aims of this study were to explore and isolate chemical constituents from the ethanol extract of Rourea induta Planch., Connaraceae, and to perform a toxicity evaluation of the extract and fractions.

\section{MATERIAL AND METHODS}

\section{Plant material}

The leaf samples from $R$. induta Planch., Connaraceae were collected in Rondonópolis city, Mato Grosso State, Brazil, in November 2007. The material was identified by Gert Hatschbach, botanist of Curitiba Botanical Museum, Brazil, and the exsiccate was registered under voucher number 261574.

\section{Extraction and isolation}

The total amount of ethanol extract was obtained from $2 \mathrm{~kg}$ of plant material in $95 \%$ ethanol, with the use of Soxhlet equipment, and then filtered, concentrated in a rotary evaporator to $300 \mathrm{~mL}$, and fractioned by liquid-liquid partition with solvents of different polarities. From this mixture, hexane, ethyl acetate and chloroform fractions were obtained. The hexane fraction was used to dissolve the filtered residue, and this was fractioned with hexane in a silica column on the Soxhlet equipment. The fractions hexane 1 and hexane 2 were obtained as a result, yielding $5.7834 \mathrm{~g}(0.289 \%)$ for hexane $1,4.9818 \mathrm{~g}(0.249 \%)$ for hexane $2,39.92 \mathrm{~g}(1.99 \%)$ for chloroform and $54.3579 \mathrm{~g}$ $(2.717 \%)$ for ethyl acetate, at the end of this procedures.
Hexane fraction 1 underwent column chromatography in silica gel stationery phase 60 Merck 0.063 $0.200 \mathrm{~mm}$, and hexane/ethyl acetate mobile phase at $5 \%$ gradient. White crystals (compound 1)(1.0314 g) were obtained among sub-fractions 1-3.

The chloroform fraction was also submitted to silica column chromatography with a hexane/ethyl acetate eluent system at 5\% gradient. It attained ethyl acetate to $100 \%$ followed by an ethyl acetate/methanol system at $5 \%$ gradient. The samples that had some crystallization were submitted to thin-layer chromatography in mobile phase with ethyl acetate, formic acid, acetic acid, water (100:11:11:27) and reactive NEU (reactive 2-aminoethylbutyrate). A pure compound (compound 2) $(0.1171 \mathrm{~g})$ was identified between sub-fractions 11-16. Sub-fractions 30-36 and 37-44 were submitted to Sephadex LH-20 with $70 \%$ methanol as the eluent, and two compounds were obtained denominated $3(0.0095 \mathrm{~g})$ and $4(0.022 \mathrm{~g})$.

During the concentration of ethyl acetate fraction, yellow crystals precipitated, which were purified by vacuum filtering and identified as compound 5 (1.5494 g).

\section{Compound identification}

The ${ }^{13} \mathrm{C}$ NMR and ${ }^{1} \mathrm{H}$ NMR spectrums were obtained on a Brucker ${ }^{\circledR}$ spectrophotometer model AC200 at $300 \mathrm{MHz}$. Ultraviolet spectroscopy was carried out on a UV-1601 Shimadzu ${ }^{\circledR}$ spectrophotometer from 200 to $450 \mathrm{~nm}$ following the Mabry, Markham \& Thomas (1970) methodology. IR spectrums were carried out on pastilles of anhydrous potassium bromide ( $\mathrm{KBr}$ ) compressed on Bomem-Hartmann \& Braum MB-series equipment and analyzed on a Biored® FTS $3500 \mathrm{GX}, 400$ to $4000 \mathrm{~cm}^{-1}$. The melting point was measured on Koffler-Reichert Austria equipment.

$N$-tetracosane (1): White crystals. mp $65^{\circ} \mathrm{C}$; IR $(\mathrm{KBr})$ vmax 2918. $2848\left(\mathrm{CH}_{2}\right) \mathrm{cm}^{-1} ;{ }^{13} \mathrm{C} \mathrm{NMR}\left(\mathrm{CDCl}_{3} ; 75\right.$ MHz) $\delta: 32.1\left(\mathrm{CH}_{2}\right) \cdot 29.9\left(\mathrm{CH}_{2}\right) \cdot 29.6\left(\mathrm{CH}_{2}\right) .22 .9\left(\mathrm{CH}_{2}\right)$. $14.3\left(\mathrm{CH}_{3}\right.$. C-1; C-24); ${ }^{1} \mathrm{H}$ NMR $\left(\mathrm{CDCl}_{3} ; 300 \mathrm{MHz}\right) 0.8$ (3H. $t$ ); 1.26 (32H. s); EIMS m/z 338 (5). $\mathrm{C}_{24} \mathrm{H}_{50}\left(\mathrm{M}^{+}\right) .44$ (100) $\left[\mathrm{C}_{3} \mathrm{H}_{7}^{+}\right] .57(95)\left[\mathrm{C}_{4} \mathrm{H}_{9}^{+}\right] .71(67)\left[\mathrm{C}_{5} \mathrm{H}_{11}^{+}\right] .85(45)$ $\left[\mathrm{C}_{6} \mathrm{H}_{13}{ }^{+}\right] .97$ (30). 113 (23). 124 (14). 141 (13). 168 (10). 194 (15). 207 (18). 267 (15). 295 (7).

Quercetin (2): Yellow amorphous powder. mp $250{ }^{\circ} \mathrm{C}$; UV $\lambda_{\max }(\log \varepsilon)(\mathrm{MeOH}) 373$ (6.32). $256(6.52)$. 266(sh) (6.19) nm; (NaOAc) 381(6.28). 325.5 (6.03), 272 (6.24), 256.5(sh) (6.29) nm; (NaOAc/ $\left.\mathrm{H}_{3} \mathrm{BO}_{3}\right) 387.5$ (6.39), $260(6.40) \mathrm{nm} ;\left(\mathrm{AlCl}_{3}\right) 458$ (6.44), $337(5.75), 271.4(6.36)$ $\mathrm{nm} ;\left(\mathrm{AlCl}_{3} / \mathrm{HCl}\right) 430$ (6.34), 363 (5.97), $265.5(6.38) \mathrm{nm}$; 
IR (KBr) vmax 3406, $3294(\mathrm{OH}), 1664(\mathrm{C}=\mathrm{O}), 1610-1400$ $(\mathrm{C}=\mathrm{C}) \mathrm{cm}^{-1} ;{ }^{13} \mathrm{C}$ NMR $\left(\mathrm{CD}_{3} \mathrm{OD} ; 75 \mathrm{MHz}\right) \delta 177.2(\mathrm{C}, \mathrm{C}-4)$, 165.3 (C, C-7), 162.3 (C, C-5), 158.1 (C, C-9), 148.5 (C, C-3'), 147.8 (C, C-2), 146 (C, C-4'), 137.1 (C, C-3), 124 (C, C-1'), $121.6(\mathrm{CH}, \mathrm{C}-6$ '), $116.1(\mathrm{CH}, \mathrm{C}-5$ '), $115.9(\mathrm{CH}$, C-2'), 104.1 (C, C-10), 99.1 (CH, C-6), $94.4(\mathrm{CH}, \mathrm{C}-8)$; ${ }^{1} \mathrm{H}$ NMR $\left(\mathrm{CD}_{3} \mathrm{OD} ; 300 \mathrm{MHz}\right) 7.72\left(1 \mathrm{H}, d, \mathrm{~J}=2.1 \mathrm{~Hz}, \mathrm{H}-2^{\prime}\right), 7.6$ $(1 \mathrm{H}, d d ; \mathrm{J}=8.7 ; 2.1 \mathrm{~Hz}, \mathrm{H}-6$ '), $6.8(1 \mathrm{H}, d, \mathrm{~J}=8.4 \mathrm{~Hz}, \mathrm{H}-5$ ' $)$, $6.36(1 \mathrm{H}, d, \mathrm{~J}=2.1 \mathrm{~Hz}, \mathrm{H}-8), 6.17(1 \mathrm{H}, d, \mathrm{~J}=2.1 \mathrm{~Hz}, \mathrm{H}-6)$.

Quercetin-3-O- $\boldsymbol{\alpha}$-arabinofuranoside (3): Yellow amorphous powder, $\mathrm{mp} 205^{\circ} \mathrm{C}$; UV $\lambda_{\max }(\log \varepsilon)(\mathrm{MeOH})$ 355 (6.46), 257 (6.57), 267(sh) (6.52) nm; (NaOAc) 370 (6.41), $271(6.58) \mathrm{nm} ;\left(\mathrm{NaOAc} / \mathrm{H}_{3} \mathrm{BO}_{3}\right) 375(6.52), 310$ (6.10), $261(6.66) \mathrm{nm} ;\left(\mathrm{AlCl}_{3}\right) 436(6.58), 275(6.66) \mathrm{nm}$; $\left(\mathrm{AlCl}_{3} / \mathrm{HCl}\right) 400$ (6.42), $270(6.60) \mathrm{nm}$; IR (KBr) vmax 3427; 3311 $(\mathrm{OH}), 1652(\mathrm{C}=\mathrm{O}), 1606,1360(\mathrm{C}=\mathrm{C}) \mathrm{cm}^{-1}$; ${ }^{13} \mathrm{C}$ NMR $\left(\mathrm{CD}_{3} \mathrm{OD} ; 75 \mathrm{MHz}\right) \delta 179.9(\mathrm{C}, \mathrm{C}-4), 166.0(\mathrm{C}$, C-7), 163.1 (C, C-5), 159.4 (C, C-9), 158.5 (C, C-2), 149.9 (C, C-3'), 146.4 (C, C-4'), 134.9 (C, C-3), 123.1 (C, C-1'), $122.9(\mathrm{CH}, \mathrm{C}-6$ ') $116.8(\mathrm{CH}, \mathrm{C}-2$ '), $116.4(\mathrm{CH}, \mathrm{C}-5$ '), 109.5 (CH, C-1"), 105.6 (C, C-10), 99.9 (CH, C-6), 94.7 (CH, C-8), 87.9 (CH, C-4"), 83.3 (CH, C-2"), $78.6(\mathrm{CH}$, C-3"), $62.5\left(\mathrm{CH}_{2}, \mathrm{C}-5\right.$ ") $)$ ' ${ }^{1} \mathrm{H}$ NMR ( $\left.\mathrm{CD}_{3} \mathrm{OD} ; 300 \mathrm{MHz}\right)$ $7.5(1 \mathrm{H}, d, \mathrm{~J}=3.3 \mathrm{~Hz}, \mathrm{H}-2$ '), $7.4(1 \mathrm{H}, l s, \mathrm{H}-6$ '), $6.9(1 \mathrm{H}$, $d, \mathrm{~J}=8.4 \mathrm{~Hz}, \mathrm{H}-5$ ') $, 6.38(1 \mathrm{H}, l s, \mathrm{H}-8), 6.2(1-\mathrm{H},, l s, \mathrm{H}-6)$, $5.58(1 \mathrm{H}, d, \mathrm{~J}=1.0 \mathrm{~Hz}, \mathrm{H}-1$ '”), $4.3(1 \mathrm{H}, d, \mathrm{~J}=4.3 \mathrm{~Hz}, \mathrm{H}-2$ '), $3.9(1 \mathrm{H}, d d, \mathrm{~J}=4.2 ; 2.4 \mathrm{~Hz}, \mathrm{H}-3$ ') $), 3.8(1 \mathrm{H}, d d, \mathrm{~J}=4.2$; $8.5 \mathrm{~Hz}, \mathrm{H}-4$ "), 3.5 (2H, m, H-5"a; H-5"b).

Quercetin-3-O- $\boldsymbol{\beta}$-xyloside (4): Yellow amorphous powder. UV $\lambda_{\max }(\log \varepsilon)(\mathrm{MeOH}) 355$ (6.65), 257 (6.74) nm; (NaOAc) 371.5 (6.58), 272.5 (6.76) nm; (NaOAc/ $\left.\mathrm{H}_{3} \mathrm{BO}_{3}\right) 375.5$ (6.70), $261(6.83) \mathrm{nm} ;\left(\mathrm{AlCl}_{3}\right) 430$ (6.68), 273 $(6.82) \mathrm{nm} ;\left(\mathrm{AlCl}_{3} / \mathrm{HCl}\right) 400(6.60), 270(6.78) \mathrm{nm} ; \mathrm{IR}(\mathrm{KBr})$ $v \max 3419,3327(\mathrm{OH}), 1651(\mathrm{C}=\mathrm{O}), 1606-1360(\mathrm{C}=\mathrm{C})$ $\mathrm{cm}^{-1},{ }^{13} \mathrm{C}$ NMR $\left(\mathrm{CD}_{3} \mathrm{OD} ; 75 \mathrm{MHz}\right) \delta 179.4(\mathrm{C}, \mathrm{C}-4), 166.0$ (C, C-7), 163.1 (C, C-5), 158.5 (C, C-2), 158.5 (C, C-9), 149.8 (C, C-3'), 146.1 (C, C-4'), 135.4 (C, C-3), 123.3 (CH, C-6'),123.0 (C, C-1'), 117.2 (CH, C-5'), 115.9 (CH, C-2'), 105.6 (C, C-10), 104.5 (CH, C-1"), 99.8 (CH, C-6), 94.7 (CH, C-8), 77.5 (CH, C-3"), 75.3 (CH, C-2"), $71.0(\mathrm{CH}$, C-4"), 67.2 ( $\left.\mathrm{CH}_{2}, \mathrm{C}-5 "\right)$; ${ }^{1} \mathrm{H} \mathrm{NMR}\left(\mathrm{CD}_{3} \mathrm{OD} ; 300 \mathrm{MHz}\right)$ $7.6(1 \mathrm{H}, \mathrm{H}-2$ '), $7.57(1 \mathrm{H}, d, \mathrm{~J}=2.1 \mathrm{~Hz}, \mathrm{H}-6$ '), $6.84(1 \mathrm{H}, s$, $\mathrm{H}-5$ '), $6.39(1 \mathrm{H}, d, \mathrm{~J}=1.8 \mathrm{~Hz}, \mathrm{H}-8), 6.16(1 \mathrm{H}, d, \mathrm{~J}=2.1 \mathrm{~Hz}$, $\mathrm{H}-6), 5.17(1 \mathrm{H}, d, \mathrm{~J}=7.2 \mathrm{~Hz}, \mathrm{H}-1$ "), $3.78(1 \mathrm{H}, d d, \mathrm{~J}=11.4$; $5.0 \mathrm{~Hz}, \mathrm{H}-5$ "'b), 3.46-3.54 (2H, $m, \mathrm{H}-3$ " e H-4"), 3.39 (1H, m, H-2"), $3.1(1 \mathrm{H}, d d, \mathrm{~J}=11.5$; 9.5Hz, H-5"a).

Quercetin-3-O- $\boldsymbol{\beta}$-galactoside (5): Yellow amorphous powder, $\mathrm{mp} 230{ }^{\circ} \mathrm{C}$; $\mathrm{UV} \lambda_{\max }(\mathrm{MeOH}) 257.9$ (6.37),
269 (sh) (6.36), 297(sh) (6.06), 359.5 (6.27) nm; ( NaOAc) 271.5 (6.30), $372.5(6.21) \mathrm{nm} ;\left(\mathrm{NaOAc} / \mathrm{H}_{3} \mathrm{BO}_{3}\right) 379.5$ (6.32), 262 (6.46) nm; $\left(\mathrm{AlCl}_{3}\right) 272$ (6.41), 424.5 (6.22) $\mathrm{nm} ;\left(\mathrm{AlCl}_{3} / \mathrm{HCl}\right) 269$ (6.40), 299.5 (sh) (6.00), 400 (6.22) $\mathrm{nm}$; IR (KBr) vmax 3429, $3244(\mathrm{OH}), 1656(\mathrm{C}=\mathrm{O}), 1606$ $1508(\mathrm{C}=\mathrm{C}) \mathrm{cm}^{-1},{ }^{13} \mathrm{C}$ NMR $\left(75 \mathrm{MHz}\right.$, DMSO-d $\left.{ }_{6}\right) \delta 177.5$ (C, C-4), 164.2 (C, C-7), 161.3 (C, C-5), 156.2 (C, C-2), 156.3 (C, C-9), 148.5 (C, C-4'), 144.9 (C, C-3'), 133.5 (C, C-3), 122.1 (CH, C-6'), 121.1 (C, C-1'), $115.9(\mathrm{CH}$, C-2'), 115.2 (CH, C-5'), 103.9 (C, C-10), 101.8 (CH, C-1"), 98.7 (CH, C-6), 93.5 (CH, C-8), 75.9 (CH, C-5"), 73.2 (CH, C3"), 71.2 (CH, C-2"), 67.9 (CH, C-4"), 60.2 $\left(\mathrm{CH}_{2}, \mathrm{C}-6 "\right)$; ${ }^{1} \mathrm{H}$ NMR (300 MHz, DMSO-d $) 12.62(1 \mathrm{H}$, $s, 5-\mathrm{OH}), 10.87(1 \mathrm{H}, s, 7-\mathrm{OH}), 9.74(1 \mathrm{H}, s, 4$ '-OH), 9.17 (1H, s, 3'-OH), 7.65 (1H, dd, J= 8.4 e $2.1 \mathrm{~Hz}, \mathrm{H}-6$ ') 7.52 $\left(1 \mathrm{H}, \mathrm{d}, J=2.1 \mathrm{~Hz}, \mathrm{H}-2^{\prime}\right), 6.82\left(1 \mathrm{H}, d, \mathrm{~J}=8.4 \mathrm{~Hz}, \mathrm{H}-5^{\prime}\right)$, $6.40(1 \mathrm{H}, d, \mathrm{~J}=2.1 \mathrm{~Hz}, \mathrm{H}-8), 6.20(1 \mathrm{H}, d, \mathrm{~J}=2.1 \mathrm{~Hz}, \mathrm{H}-6)$, $5.38(1 \mathrm{H}, d, J=8.4 \mathrm{~Hz}, \mathrm{H}-1$ " $), 5.14(1 \mathrm{H}, s, 2$ " $-\mathrm{OH}), 4.87$ (1H, s, 3"-OH), $4.44(2 \mathrm{H}, s, 4 ", 6 "-\mathrm{OH})$.

\section{Brine shrimp bioassay}

The brine shrimp (Artemia salina) method was first described by Meyer et al. (1982). The eggs of brine shrimp hatched within 48 hours in artificial sea water, producing a large number of larvae. The samples, ethanolic extract, and all the fractions, were tested at concentrations of 1000 , 100 and $10 \mu \mathrm{g} / \mathrm{mL}$. Ten shrimps were transferred to the samples and after 24 hours survivors were counted and percentage of deaths at each dose recorded. Quinidine sulfate was used as a positive control and the solvent used to prepare the samples as the negative control. The test was run in triplicate and the data was analyzed by the Probitos method to determine $\mathrm{LD}_{50}$ with a $95 \%$ confidence interval.

\section{Hemolytic activity}

Ethanolic extract and fractions were submitted to hemolytic activity tests. The first method tested was based on the WHO (1998) and Carvalho et al. (2009). For this method, a $2 \%$ sheep red blood cell suspension (Newprov ${ }^{\circledR}$ ) was prepared in $\mathrm{pH} 7.4$ phosphate buffer. The samples were diluted in $10 \%$ ethanol with $\mathrm{NaCl} 0.9 \%$ and tested at concentrations of $1000,500,200$ and $100 \mu \mathrm{g} / \mathrm{mL}$. A volume of $1.0 \mathrm{~mL}$ of the blood suspension was added to the samples which were then mixed slowly and left to rest for 30 minutes, mixed again and kept at rest for a further 150 minutes. Subsequently, the samples were centrifuged for 5 minutes at $3000 \mathrm{rpm}$. Distilled water was used as a positive control and solvent and phosphate buffer as a negative control. The result was reported as presence or 
absence of hemolysis based on the supernatant tonality after centrifugation. The presence of a red blood cell precipitate indicated a negative result.

The second method tested was agar diffusion using plates with sheep red blood cell agar $\left(\mathrm{Newprov}^{\circledR}\right)$. The samples were prepared at $1000 \mu \mathrm{g} / \mathrm{mL}$ and impregnated in sterile discs (Whatmann $\mathrm{n}^{\circ} 1$, diameter $7 \mathrm{~mm}$ ). After solvent evaporation, the discs were distributed in the plates, which were incubated at $36^{\circ} \mathrm{C}$ for 24 hours. Hemolytic halos were then measured and results expressed as mean halos found in duplicate. Discs with the solvents used for sample dilution were used as a negative control and Triton $\mathrm{X}-100$ at $1000 \mu \mathrm{g} / \mathrm{mL}$ as a positive control (Efing, 2008).

\section{RESULTS AND DISCUSSION}

The hexane fraction of ethanol extract from the leaves of $R$. induta was subjected to a chromatographic step over silica gel to yield compound $\mathbf{1}$ (n-tetracosane), while the chloroform fraction was subject to multiple chromatographic steps over silica gel to yield compounds 2, 3 and 4 (quercetin, quercetin-3- $O$ - $\alpha$-arabinofuranoside and quercetin-3-O- $\beta$-xyloside, respectively). Compound 5 (quercetin-3-O- $\beta$-galactoside) was obtained during ethyl acetate fraction concentration.

The ${ }^{1} \mathrm{H}$ NMR signals for compound $\mathbf{1}$, in the range $0.8-1.7 \mathrm{ppm}$, indicated a region characteristic of aliphatic alicyclic compounds with three hydrogen at $\delta 0.8$, whereas the signal integration at $\delta 1.26$ indicated about 32 hydrogen, corresponding to $\mathrm{CH}_{2}$. The ${ }^{13} \mathrm{C}$ NMR spectrum characterized five signals in the range $14-32 \mathrm{ppm}$. The mass spectrum showed a characteristic fragmentation of aliphatic hydrocarbons, with 14 mass units between peaks. The fragmentation standard is determined by C-C fragmentation because hydride (H-) elimination is difficult. The spectrum showed higher intensity peaks (44 and 57) that represented the fragments with three and four carbons, and a low intensity molecular ion $(\mathrm{m} / \mathrm{z})$ of 338 , characteristic of long chain. The melting point was $65^{\circ} \mathrm{C}$ a typical value for a hydrocarbon. These results were in line with those of Siddiqui et al. (2004) and denoted that compound 1 was n-tetracosane $\left(\mathrm{C}_{24} \mathrm{H}_{50}\right)$.

For compounds 2-5, the UV spectrum showed typical results for flavonoids, with bands between $256-259 \mathrm{~nm}$ relative to ring A and 348-360 relative to ring B (Mabry, Markham, Thomas, 1970), which suffers a hypsochromic effect in comparison to quercetin due to C-3 substitution for compounds 3, 4 and $\mathbf{5}$ (Santos, Schripsema, Kuster, 2005). For all samples, the addition of $\mathrm{NaOAc}$ produced a bathochromic effect of band II, which indicated a free hydroxyl in position $7 . \mathrm{H}_{3} \mathrm{BO}_{3}$ led to a bathochromic shift of band I in the presence of an orthodihydroxyl group in ring $\mathrm{B}$. For 3, 4 and 5, the spectrum with $\mathrm{HCl}$ revealed a bathochromic shift for bands I and II. The addition of $\mathrm{AlCl}_{3}$ caused a bathochromic effect of band I between $35-55 \mathrm{~nm}$ compared with methanol spectrum, typically shifted for 5 -hydroxyflavons with position 3 replaced. For compound 2 this effect was $57 \mathrm{~nm}$, a typical result for 3,5-hydroxyflavons (Mabry, Markham, Thomas, 1970).

The infrared spectrum revealed two absorption bands in 3430 and $3300 \mathrm{~cm}^{-1}$ that typically result from the $\mathrm{OH}$ link axial deformation. Moreover, the existence of an aromatic ring may be observed, evidenced by a set of bands that range between 1600 and $1400 \mathrm{~cm}^{-1}$, which denotes the axial deformation of the aromatic $\mathrm{C}=\mathrm{C}$. Bands close to $1650 \mathrm{~cm}^{-1}$ suggest the presence of carbonyl (Silverstein, Bassler, Morril, 1994).

The ${ }^{13} \mathrm{C}$ NMR signals for these compounds were in good agreement with those of quercetin, the difference between them was located at the glycoside linked to the $\mathrm{C}-3$ of the aglycone. This glycoside was identified by the signals at sugar region $(60-80 \mathrm{ppm})$ in the spectrum by comparing against those described in the literature. For compound 2, no signals were identified at this region (Andersen, Markham, 2006). The signals for compound 3 indicated arabinofuranoside (Vvdenskaya et al., 2004), for compound 4 xyloside (Yan et al., 2002) and for the isolated 5 galactoside (Jiang et al., 1990). The ${ }^{1} \mathrm{H}$ NMR signals for these compounds were found in the aromatic region (6-8ppm) and sugar region (3-4.2 ppm). The configuration of the sugars were determined by the coupling constant of the anomeric hydrogen, where compound $\mathbf{3}$ showed the anomeric hydrogen at $\delta 5.58$ with $\mathrm{J}=1.00 \mathrm{~Hz}$, indicating that arabinofuranoside is $\alpha$ (Zhang et al., 2005), compound 4 was $\delta 5.17$ with $\mathrm{J}=7.2 \mathrm{~Hz}$, showing a $\beta$ configuration for xyloside (Yan et al., 2002), and compound $\mathbf{5}$ was $\delta 5.39$ with $\mathrm{J}=7.8 \mathrm{~Hz}$, a $\beta$ configuration for galactoside (Santos, Schripsema, Kuster, 2005).

After the above analysis the results were compared against literature data (Tables I, II and III) and compound

TABLE I - Compound 1 NMR (ppm) experimental data compared against literature data

\begin{tabular}{cc}
\hline $\begin{array}{c}\text { Experimental } \\
{ }^{13} \mathbf{C}\end{array}$ & $\begin{array}{c}\text { Siddiqui } \text { et al. (2004) } \\
{ }^{13} \mathbf{C}\end{array}$ \\
\hline 32.1 & 32.1 \\
29.9 & 29.8 \\
29.6 & 29.5 \\
22.9 & 22.8 \\
14.3 & 14.1 \\
\hline
\end{tabular}


TABLE II - Compounds 2 and 3 NMR (ppm) experimental data compared against literature data

\begin{tabular}{|c|c|c|c|c|c|c|c|}
\hline \multirow[t]{3}{*}{ Position } & \multicolumn{3}{|c|}{ Quercetin } & \multicolumn{4}{|c|}{ Quercetin-3- $O$ - $\alpha$-arabinofuranoside } \\
\hline & \multicolumn{2}{|c|}{ Experimental } & \multirow{2}{*}{$\begin{array}{c}\text { Andersen, } \\
\text { Markham (2006) } \\
{ }^{13} \mathrm{C}\end{array}$} & \multicolumn{2}{|c|}{ Experimental } & \multicolumn{2}{|c|}{ Lhuillier (2007) } \\
\hline & ${ }^{13} \mathrm{C}$ & ${ }^{1} \mathrm{H}$ & & ${ }^{13} \mathrm{C}$ & ${ }^{1} \mathrm{H}$ & ${ }^{13} \mathrm{C}$ & ${ }^{1} \mathrm{H}$ \\
\hline 2 & 147.8 & - & 148.00 & 158.5 & - & 158.2 & - \\
\hline 3 & 137.1 & - & 137.21 & 134.9 & - & 133.7 & - \\
\hline 4 & 177.2 & - & 177.33 & 179.9 & - & 178.8 & - \\
\hline 5 & 162.3 & - & 162.50 & 163.1 & - & 161.8 & - \\
\hline 6 & 99.1 & $\begin{array}{c}6.17 d \\
(J=2.1 \mathrm{~Hz})\end{array}$ & 99.25 & 99.9 & 6.2 ls & 98.9 & $\begin{array}{c}6.21 d \\
(J=2.0 \mathrm{~Hz})\end{array}$ \\
\hline 7 & 165.3 & & 165.34 & 166.0 & - & 164.8 & - \\
\hline 8 & 94.4 & $\begin{array}{c}6.36 d \\
(J=2.1 \mathrm{~Hz})\end{array}$ & 94.40 & 94.7 & $6.38 l s$ & 93.5 & $\begin{array}{c}6.4 d \\
(J=2.0 \mathrm{~Hz})\end{array}$ \\
\hline 9 & 158.1 & - & 158.22 & 159.4 & - & 157.4 & - \\
\hline 10 & 104.1 & - & 104.52 & 105.6 & - & 104.4 & - \\
\hline 1 ' & 124.0 & - & 124.15 & 123.1 & - & 121.9 & - \\
\hline 2 ' & 115.9 & $\begin{array}{c}7.72 \mathrm{~d} \\
(J=2.1 \mathrm{~Hz})\end{array}$ & 115.99 & 116.8 & $\begin{array}{c}7.5 d \\
(J=3.3 \mathrm{~Hz})\end{array}$ & 115.7 & $\begin{array}{c}7.54 d \\
(J=2.1 \mathrm{~Hz})\end{array}$ \\
\hline $3^{\prime}$ & 148.5 & - & 148.75 & 149.9 & - & 148.7 & - \\
\hline $4^{\prime}$ & 146.0 & - & 146.21 & 146.4 & - & 145.1 & - \\
\hline 5 & 116.1 & $\begin{array}{c}6.8 d \\
(J=8.4 \mathrm{~Hz})\end{array}$ & 116.22 & 116.4 & $\begin{array}{c}6.9 d \\
(J=8.4 \mathrm{~Hz})\end{array}$ & 115.3 & $\begin{array}{c}6.92 d \\
(J=8.3 \mathrm{~Hz})\end{array}$ \\
\hline \multirow[t]{2}{*}{$6^{\prime}$} & 121.6 & $\begin{array}{c}7.6 d d \\
(J=8.7 ; 2.1 \mathrm{~Hz})\end{array}$ & 121.67 & 122.9 & $7.4 l_{s}$ & 121.8 & $\begin{array}{c}7.5 d d \\
(J=8.3 \text { e } 2.1 \mathrm{~Hz})\end{array}$ \\
\hline & & & & \multicolumn{4}{|c|}{ Arabinofuranoside } \\
\hline $1 "$ & - & - & - & 109.5 & $5.5 s$ & 108.5 & $\begin{array}{c}5.48 d \\
(J=1.0 \mathrm{~Hz})\end{array}$ \\
\hline $2 "$ & - & - & - & 83.3 & $\begin{array}{c}4.3 d \\
(J=2.4 \mathrm{~Hz})\end{array}$ & 82.1 & $\begin{array}{c}4.35 d d \\
(J=3.0 ; 1.0 \mathrm{~Hz})\end{array}$ \\
\hline $3 "$ & - & - & - & 78.6 & $\begin{array}{c}3.9 d d \\
(\mathrm{~J}=4.2 ; 2.4 \mathrm{~Hz})\end{array}$ & 77.5 & $\begin{array}{c}3.93 d d \\
(J=5.0 ; 3.0 \mathrm{~Hz})\end{array}$ \\
\hline $4 "$ & - & - & - & 87.9 & $\begin{array}{c}3.8 d d \\
(\mathrm{~J}=4.2 ; 8.5 \mathrm{~Hz})\end{array}$ & 86.8 & $3.89 \mathrm{~m}$ \\
\hline $5 "$ & - & - & - & 62.5 & $3.5 \mathrm{~m}(2 \mathrm{H})$ & 61.4 & $\begin{array}{c}3.52 d d \\
(J=11.5 ; 4.0 \mathrm{~Hz}) \\
3.48 d d \\
(J=11.5 ; 3.6 \mathrm{~Hz})\end{array}$ \\
\hline
\end{tabular}

Note: $l s=$ large singlet, $d=$ doublet, $d d=$ double doublet, $m=$ multiplet, $J=$ coupling constant

2 was identified as quercetin (Andersen, Markham, 2006), 3 as quercetin-3- $O$ - $\alpha$-arabinofuranoside (Vvdenskaya et al., 2004; Lhuillier, 2007), 4 as quercetin-3-O- $\beta$-xyloside (Yan et al., 2002), and $\mathbf{5}$ as quercetin-3-O- $\beta$-galactoside (Jiang et al., 1990), also known as hyperin (Figure 1).

Quercetin and quercetin-3- $O$ - $\alpha$-arabinofuranoside were previously isolated from the Connaraceae family spe- cies Byrsocarpus coccineus (Ahamadu et al., 2007), while hyperin was previously isolated in the Rourea microphylla species (Jiang et al., 1990). However, this is the first time these have been isolated in the $R$. induta species. There are no previous reports on the isolation of quercetin-3-O$\beta$-xyloside in the Connaraceae family.

The toxicity evaluation against Artemia salina 
TABLE III - Compounds 4 and 5 NMR (ppm) experimental data compared against literature data

\begin{tabular}{|c|c|c|c|c|c|c|c|c|}
\hline \multirow{3}{*}{ Position } & \multicolumn{4}{|c|}{ Quercetin-3-O-xyloside } & \multicolumn{4}{|c|}{ Quercetin-3-O- $\beta$-galactoside } \\
\hline & \multicolumn{2}{|c|}{ Experimental } & \multicolumn{2}{|c|}{ Yan et al. (2002) } & \multicolumn{2}{|c|}{ Experimental } & \multicolumn{2}{|c|}{ Jiang et al. (1990) } \\
\hline & ${ }^{13} \mathrm{C}$ & ${ }^{1} \mathrm{H}$ & ${ }^{13} \mathrm{C}$ & ${ }^{1} \mathrm{H}$ & ${ }^{13} \mathrm{C}$ & ${ }^{1} \mathrm{H}$ & ${ }^{13} \mathrm{C}$ & ${ }^{1} \mathrm{H}$ \\
\hline 2 & 158.5 & - & 152.0 & - & 156.2 & - & 156.28 & - \\
\hline 3 & 135.4 & - & 130.0 & - & 133.5 & - & 133.61 & - \\
\hline 4 & 179.4 & - & 179.5 & - & 177.5 & - & 177.44 & - \\
\hline 5 & 163.1 & - & 162.0 & - & 161.3 & - & 161.15 & - \\
\hline 6 & 99.8 & $\begin{array}{c}6.16 d \\
(J=2.1 \mathrm{~Hz})\end{array}$ & 100.1 & 6.20 & 98.7 & $\begin{array}{c}6.2 d \\
(J=1.8 \mathrm{~Hz})\end{array}$ & 98.62 & $6.20 s$ \\
\hline 7 & 166.0 & - & 166.3 & - & 164.2 & - & 164.02 & - \\
\hline 8 & 94.7 & $\begin{array}{c}6.39 d \\
(J=1.8 \mathrm{~Hz})\end{array}$ & 94.9 & 6.39 & 93.5 & $\begin{array}{c}6.4 d \\
(J=2.1 \mathrm{~Hz})\end{array}$ & 93.45 & $6.41 s$ \\
\hline 9 & 158.5 & - & 158.6 & - & 156.3 & - & 156.28 & - \\
\hline 10 & 105.6 & - & 100.1 & - & 103.9 & - & 103.94 & - \\
\hline 1 ' & 123.0 & - & 123.4 & - & 121.1 & - & 121.16 & - \\
\hline 2 ' & 115.9 & 7.6 & 116.2 & 7.6 & 115.9 & $\begin{array}{c}7.5 d \\
(J=2.4 \mathrm{~Hz})\end{array}$ & 115.17 & $7.53 s$ \\
\hline $3^{\prime}$ & 149.8 & - & 143.0 & - & 144.9 & - & 144.67 & - \\
\hline $4^{\prime}$ & 146.1 & - & 146.0 & - & 148.5 & - & 148.32 & - \\
\hline 5 & 117.2 & $6.84 s$ & 117.4 & 6.85 & 115.2 & $\begin{array}{c}6.8 d \\
(J=8.7 \mathrm{~Hz})\end{array}$ & 116.10 & $\begin{array}{c}6.82 d \\
(J=8 \mathrm{~Hz})\end{array}$ \\
\hline \multirow[t]{2}{*}{$6^{\prime}$} & 123.3 & $\begin{array}{c}7.57 \mathrm{~d} \\
(J=2.1 \mathrm{~Hz})\end{array}$ & 123.2 & 7.57 & 122.1 & $\begin{array}{c}7.65 d d \\
(J=8.4 ; 2.1 \mathrm{~Hz})\end{array}$ & 121.62 & $\begin{array}{c}7.67 d \\
(J=8 \mathrm{~Hz})\end{array}$ \\
\hline & \multicolumn{4}{|c|}{ Xylose } & \multicolumn{4}{|c|}{ Galactoside } \\
\hline $1 "$ & 104.5 & $\begin{array}{c}5.17 d \\
(J=7.2 \mathrm{~Hz})\end{array}$ & 104.7 & $\begin{array}{c}5.18 d \\
(J=6 \mathrm{~Hz})\end{array}$ & 101.8 & $\begin{array}{c}5.39 d \\
(J=7.8 \mathrm{~Hz})\end{array}$ & 102.12 & \\
\hline $2 "$ & 75.3 & $3.39 \mathrm{~m}$ & 75.4 & $3.4 d$ & 71.2 & $\begin{array}{c}3.55 d t \\
(J=7.8 ; 3.9 \mathrm{~Hz})\end{array}$ & 71.26 & \\
\hline $3 "$ & 77.5 & $3.46-3.54$ & 77.7 & $3.34 \mathrm{~s}$ & 73.2 & 3.33 & 73.27 & \\
\hline $4 "$ & 71.0 & $3.46-3.54$ & 71.2 & $3.45-3.54 m$ & 67.9 & $3.64 l s$ & 67.93 & \\
\hline $5 "$ & 67.2 & $\begin{array}{c}3.1 d d \\
(J=11.5 ; 9.5 \mathrm{~Hz}) \\
3.78 d d \\
(J=11.4 ; 5.0 \mathrm{~Hz})\end{array}$ & 67.4 & $\begin{array}{l}3.10 d d \\
3.76 d d\end{array}$ & 75.9 & $3.30 s$ & 75.73 & \\
\hline $6 "$ & - & - & - & - & 60.2 & 3.25 e $3.45(2 \mathrm{H})$ & 60.14 & \\
\hline
\end{tabular}

Note: $s=$ singlet; $l s=$ large singlet, $d=$ doublet, $d d=$ double doublet, $d t=$ double triplet, $m=$ multiplet, $J=$ coupling constant

(Table IV) demonstrated that all the samples showed a median lethal concentration greater than $1000 \mu \mathrm{g} / \mathrm{mL}$, the maximum concentration for a sample to be considered active (Meyer et al., 1982).

This bioassay is used to evaluate the acute toxicity and is considered essential as a preliminary assay in the study of compounds with potential biological activities (Amaral, Silva, 2008). Analyzing the results, it can be concluded that neither the ethanolic extract, nor the fractions from $R$. induta exhibited toxic potential against the brine shrimp.

For the hemolytic tests, the tested sample showed no hemolytic ability according to the two methods analyzed. In the test using the red blood cell suspension, none of the supernatants showed red coloration and all had a red blood cell precipitate at the tested doses and therefore caused no hemolysis in red blood cells. This result indicates that neither the fractions nor the ethanolic extract are toxic against red blood cells. These results were confirmed by the agar 
TABLE IV - Artemia salina lethality and $\mathrm{LD}_{50}$

\begin{tabular}{lccccc}
\hline Sample & \multicolumn{3}{c}{ Mortality/Concentration } & $\mathrm{LD}_{50}(\mu \mathrm{g} / \mathrm{mL})$ & $\begin{array}{c}95 \% \text { Confidence } \\
\text { interval }(\mu \mathrm{g} / \mathrm{mL})\end{array}$ \\
\hline Ethanolic extract & $10(\mu \mathrm{g} / \mathrm{mL})$ & $100(\mu \mathrm{g} / \mathrm{mL})$ & $1000(\mu \mathrm{g} / \mathrm{mL})$ & $>1000$ & - \\
Fraction Hexane 1 & 1 & 1 & 4 & $>1000$ & - \\
Fraction Hexane 2 & 0 & 0 & 0 & $>1000$ & - \\
Fraction Chloroform & 0 & 1 & 4 & $>1000$ & - \\
Fraction Ethyl Acetate & 0 & 0 & 0 & $>1000$ & - \\
Quinidine sulfate & 16 & 0 & 1 & 50.12 & $35.80-70.16$ \\
\hline
\end{tabular}

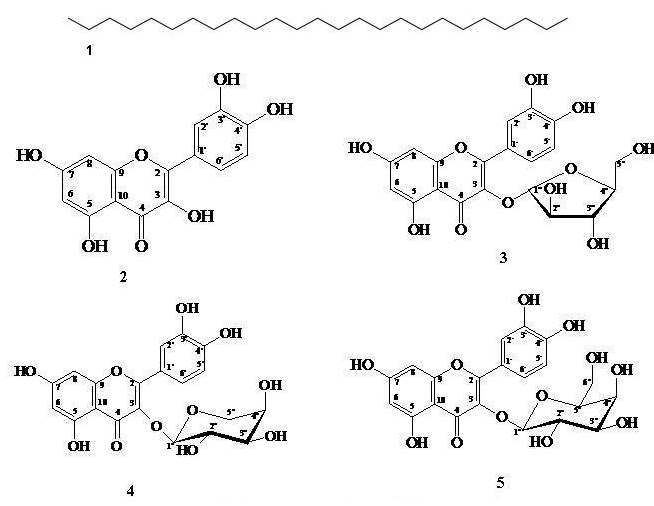

FIGURE 1- Chemical structures of compounds 1-5.

diffusion method, on which none of the samples had a hemolytic halo around the impregnated disc, confirming the absence of hemolytic activity. Similarly, the negative controls exhibited no hemolytic halo, whereas the Triton $\mathrm{X}-100$ had a halo of $2 \mathrm{~cm}$.

Red blood cell lyses can cause an elevation in plasma hemoglobin leading to harmful effects in the kidneys (nephrotoxicity), and in the cardiovascular system (vasomotor effect) (Carvalho et al., 2007). Therefore, negative hemolytic activity is a good indicator because it confirms no toxicity to erythrocyte membrane.

The acute toxicity results showed that the Rourea induta extracts had no toxic potential, thus favoring the species for further study such as pharmacological analyses, to determine therapeutic properties of this species.

\section{ACKNOWLEDGEMENTS}

We would like to thank Gert Hatschbach, botanist of Museu Botânico de Curitiba, for identifying the species, and the Universidade Estadual de Maringá for the NMR spectrums.

\section{REFERENCES}

AHAMADU, A. A.; HASSAN, H. S.; ABUBAKAR, M. U.; AKPULU, N. Flavonoid Glycosides from Byrsocarpus coccineus Leaves. Schum. and Thonn (Connaraceae). Afr. J. Trad.C.A.M., v.4, n.3, p.257-260, 2007.

AMARAL, E.A.; SILVA, R.M.G. Avaliação da toxicidade aguda de angico (Anadenanthera falcata), pau-santo (Kylmeyera coreaceae), aroeira (Myracrodruon urundeuva) e cipóde-são-jõao (Pyrostegia venusta), por meio do bioensaio com Artemia salina. Perquirere, Rev. Eletr. da Pesquisa. Avaiable at: $<$ http://www.unipam.edu.br/perquirere/file/ file/2008_cb/artigo_eni.pdf $>$. Accessed on: April, 2010.

ANDERSEN, O.M.; MARKHAM, K.R. Flavonoids, chemistry, biochemistry and applications. New York: CRC Press; 2006. $1197 \mathrm{p}$.

CARVALHO, E.B.; BORGES, E.L.; CARLOS, L.M.B.; SILVA, M.A.M; MAGALHÃES, S.M.M; GOMES, F.V.B.A.F.; CARVALHO, M.J.C.; QUIXADÁ, A.T.S.; PITOMBEIRA, M.H.S. Efeito da bomba de infusão de soluções sobre o grau de hemólise em concentrado de hemácias. Rev. Bras. Hematol. Hemoter., v.29, n.2, p.149-152, 2007.

CARVALHO, M.S.; MORAIS-COSTA, F.; CARDOSO-FILHO, O.; FERREIRA, B.C.; CARVALHO, S.; SILVA, W.A.; OLIVEIRA, D.A. Estudo preliminar da fitotoxicidade de Myracrodrum urundeuva fr. Allemao (Anacardiaceae). In: CONGRESSO DE ECOLOGIA DO BRASIL, 9., São Lourenço, 2009. Anais. São Lourenço: SEB, 2009. p.1-2.

EFING, L.M.A.C. Compostos bioativos do material resinoso, subproduto do processamento da erva-mate (Ilex paraguariensis A.St.-Hil.). Curitiba, 2008. 108 f. [Thesis of PhD degree in Food Technology. Federal University of Paraná]. 
FONSECA, L.C.M.; PROENÇA, C.E.B. Connaraceae. In: CAVALCANTI, T.B.; RAMOS, A.E. Flora do Distrito Federal. 2.ed. Brasília: Embrapa Recursos Genéticos e Biotecnologia, 2002. p.41-47.

FORERO, E. Uma nueva especie mexicana de Rourea (Connaraceae). Caldasia, v.29, n.1, p.19-21, 2007.

FORERO, E. A revision of american species of Rourea Subg. Rourea (Connaraceae). Mem. New York Bot. Gard., v.26, n.1, p.1-119, 1976.

JIANG, J-Q.; FANG, S-D.; XU, C-S.; LUO, J-T. Studies on the chemical constituents of Rourea microphylla (Hook. Et Arn) Planch. Acta Bot. Sin., v.32, n.5, p.376-379, 1990.

LHUILLIER, M.A. Contribution a l'etude phytochique de quatre plantes malgaches: Agauri salicifolia HOOK.F ex Oliver, Agauria polyphylla Baker (Ericaceae), Tambourissa trichophyla Baker (Monimiaceae) et Embelia concinna Baker (Myrsinaceae). Toulouse, 2007. $214 \mathrm{f}$. [Thesis of $\mathrm{PhD}$ degree. L'institut National Polytechnique de Toulouse. Sciences des Agroressources, École doctorale: Transferts, Dynamique des Fluides, Energie et Procédés].

LORENZI, H.; SOUZA, V.C. Botânica sistemática: guia ilustrado para identificação das famílias de angiospermas da flora brasileira, baseado em APG II. Nova Odessa: Instituto Plantarum, 2005. 640 p.

LENZA, E.; FERREIRA, J. N.; CONSOLARO, H.; AQUINO, F.G. Biologia reprodutiva de Rourea induta Planch. (Connaraceae), uma espécie heterostílica de cerrado do Brasil Central. Rev. Bras. Bot.,v.31, n.3, p.389-398, 2008.

MABRY, T. J. MARKHAM, K.R.; THOMAS, M.B. The systematic identification offlavonoids. New York: SpringerVerlag, 1970. 354 p.

MEYER, B.N.; FERRIGNI, N.R.; PUTNAM, J.E.; JACOBSEN, L.B.; NICHOLS, D.E; MCLAUGHLIN, J.L. Brine shrimp: a convenient general bioassay for active plant constituents. Planta Med., v.45, n.1, p.31-34, 1982.
OLIVEIRA, V.M.A.; CARNEIRO, A.L.B.; CAUPER, G.S.B.; POHLIT, A.M. In vitro screening of amazonian plants for hemolytic activity and inhibition of platelet aggregation in human blood. Acta Amaz., v.39, n.4, p.973-980, 2009.

RECOR. Reserva ecológica do IBGE. O cerrado brasileiro. Available at: <http://www.recor.org.br/cerrado/cerrado. html>. Accessed on: 07 fev. 2009.

SANTOS, P.M.L. SCHRIPSEMA, J.; KUSTER, R.M. Flavonóides $O$-glicosilados de Croton campestris St. Hill. (Euphorbiaceae). Rev. Bras. Farmacogn., v.15, n.4, p.321$325,2005$.

SIDDIQUI, B. S.; RASHEED, M.; ILYAS, F.; GULZAR, T.; TARIQ, R. M.; NAQVI, S. N.H. Analysis of Insecticidal Azadirachta indica A. Juss. Fractions. Z. Naturforsch., v.59c, n.1/2, p.104-112, 2004.

SILVERSTEIN, R.M.; BASSLER, F.X.; MORRIL, T.C. Identificação espectrométrica de compostos orgânicos. 5.ed. Rio de Janeiro: Guanabara Koogan, 1994. 387 p.

VVDENSKAYA, I. O.; ROSEN, R. T.; GUIDO, J. E.; RUSSEL, D. J.; MILLS, K. A.; VORSA, N. Characterization of Flavonols in Cranberry (Vaccinium macrocarpon) Powder. J. Agric. Food Chem., v.52, n.2, p.188-195, 2004.

WORLD HEALTH ORGANIZATION. Quality control methods for medicinal plant materials. Geneve, 1998. 127 p.

YAN, X.; MURPHY, B. T.; HAMMOND, G. B.; VINSON, J. A.; NETO, C. C. Antioxidant activities and antitumor screening of extracts from cranberry fruit (Vaccinium macrocarpon). J. Agric. Food Chem., v.50, n.21, p.5844-5849, 2002.

ZHANG, X.; THUONG, P.T.; WENYI, J.; SU, N.D.; DAIEUN, S.; KIHAWAN, B.; SIK, K.S. Antioxidant activity of anthraquinones and flavonoids from flower of Reynoutria sachalinensis. Arch. Pharmacal Res., v.28, n.1, p.22-27, 2005.

Received for publication on $7^{\text {th }}$ April 2010 Accepted for publication on $15^{\text {th }}$ October 2010 